\title{
A serialization based partial decoupling approach for multidisciplinary design optimization of complex systems
}

\author{
Wenqiang Yuan ${ }^{1}$, Yusheng Liu ${ }^{1 *}$, Hongwei Wang ${ }^{2}$, Xiaoping $\mathrm{Ye}^{3}$ \\ (1. State Key Lab. of CAD\&CG, Zhejiang University, Hangzhou, China, 310058) \\ (2. School of Engineering, University of Portsmouth, Portsmouth, UK, PO1 3DJ) \\ (3. Institute of Lishui, Lishui, 323000)
}

\begin{abstract}
The optimization for multidisciplinary engineering systems is highly complicated, which involves the decomposing of a system into several individual disciplinary subsystems for obtaining optimal solutions. Managing the coupling between subsystems remains a great challenge for global optimization as existing methods involve inefficient iterative solving processes and thus have higher time cost. Some strategies such as discipline reorder, coupling suspension and coupling ignoring, can to some extent reduce the execution cost. However, these approaches also have some drawbacks such as low search efficiency because of overwhelmed constraints at the system level and huge iterative computational cost for maintaining system consistency. More important issues should be considered including maintaining the whole system compatibility when achieving minimization/maximization of objective functions, ensuring a balance of running times between the solving processes for different disciplines and determining which discipline should be resolved to achieve a better performance. To overcome the above drawbacks, a Serialization based Partial Decoupling (SPD) approach is proposed in this study, which consists of three main steps. Firstly, different disciplines are clustered into some subsystems by analyzing the interdisciplinary sensitivities. Then, for each subsystem a serialization process is proposed to ensure no coupling loops exist and the subsystem can be solved with no iteration, which can reduce the time cost for solving the disciplinary problem to a large degree. Finally, a local optimization model is constructed for each subsystem to maintain the scale of the global optimizer and ensure mutual independence and parallel processing. The proposed three-layer framework ensures the feasibility of solving for each subsystem and improves the efficiency of optimization execution. Several experiments have been conducted to demonstrate the effectiveness and feasibility of the proposed approach.
\end{abstract}

Keywords: Serialization; Partial decoupling; Clustering analysis; Serializable solving; Local optimization; MDO

\section{Introduction}

The design problems in modern engineering systems generally have a large scale and involve many factors from a number of domains. Therefore, this type of problem is often logically divided into several disciplines by researchers and engineers, each of which is derived from one or more major domains [1-3]. An imperative issue in the case of multidisciplinary design and optimization is to address the unavoidable couplings among these disciplines [4-6]. Thus, the complex couplings and dependencies should be considered to get the optimal solutions during the process of complex system design [7-9]. With the requirements of high computational precision for highly complex design problems, the traditional theories of complex system design have evident limitations as they have no consideration of interdisciplinary couplings 
have shown limitations. Multidisciplinary Design Optimization (MDO) [10-12] is a hot research field in recent years in which the interdisciplinary couplings are considered to construct high-fidelity models of complex problems during the early analysis phase [13-15]. However, how to solve MDO problems efficiently is still a great challenge. A lot of research has been conducted to develop efficient and feasible decoupling strategies for MDO problems [16-17].

Several general-purpose optimization methods have been proposed for MDO problems in engineering [18-19]. Sobieski did pioneering working in this field and proposed a solving method based on sensitivity [20]. The core idea is to consider the effects among the disciplines concerned at each time of iteration. Several years later, a new architecture was proposed based on the concept of subspace. These two ideas are considered as the foundation of this field. The current state-of-the-art work related to the MDO architecture can be classified into two main types, namely single-level optimizer and bi-level optimizers. The former contains multidisciplinary feasible (MDF), individual discipline feasible (IDF) and all at once (AAO) .

Specifically, MDF is a basic architecture, which consists of a global optimizer and a uniform system solver. The optimizer manages optimization task while the solver is responsible for analyzing the disciplines involved in the solving process. The global optimizer provides values of design variables that are used as the inputs for the solver. The coupling variables in the system are solved through lots of complete iterative computations. This architecture does not change the global optimization model. However, a complete interdisciplinary analysis is required and the computational cost is generally expensive. IDF avoids the iterative interdisciplinary analysis by adding the coupling variables as global design variables and some constraints of the global optimization model. The result is that the search space has been decreased and the computation time of the global optimizer has increased. AAO is based on IDF and further decreases the complexity of the analysis process. The state variables in different disciplines are also added into the global optimizer and thus the search space is further decreased. The architectures with two levels of optimizers based on decomposition strategy contain collaborative optimization (CO) [21], concurrent system synthesis optimization (CSSO) [22] and bi-level integrated system synthesis (BLISS) [23]. The complex optimization problems are hierarchically decomposed into several sub-problems when these approaches are adopted, each of which constructs a local optimizer to deal with its corresponding sub-problems and processes its own design variables and constraints. The main problems of two-level optimizer architectures include: (1) the local optimizers are not synchronous and thus the global optimizer cannot be executed unless all the local optimizers are completed, which affects the whole optimization performance; (2) data should be exchanged between the global optimizer and all of the local optimizers, and thus a lot of data exchange operations are needed; (3) the statistical techniques and gradient information are required, which is a great challenge for constructing an efficient and effective local optimization model for a complex multidisciplinary system.

It can be seen from the above analysis that even though considerable work has been done on MDO, there still exist some deficiencies for the existing approaches. Firstly, they emphasize reorganizing the disciplines by considering the degree of coupling. With regard to a multidisciplinary system consisting 
of a large number of disciplines, the degrees of coupling between different disciplines are quite different. It is not reasonable to decompose them in a uniform way. Secondly, for the tightly-coupled disciplines with some bi-directional couplings, decoupling them completely may lead to increased computation. Lastly, computational burden of the global optimizer is heavy as it needs process too much information. In this sense, how to handle the decoupling variables with a localized way in which only the corresponding subsystem is related should be considered.

In this study, a serialization based partial decoupling (SPD) method is proposed to tackle the decoupling problem. The process of SPD method mainly consists of three steps. Firstly, several disciplines of a system are clustered into some subsystems based on the coupling sensitivity, i.e., each subsystem is made up of several disciplines. Secondly, a serialization operation is executed for partial decoupling between subsystems by removing the coupling loops in an efficient manner. Finally, a local optimization model is constructed and executed to maintain the consistency of the decoupling variables for each subsystem. The most primary mechanism of SPD is that only a small percentage of coupling variables are required to be decoupled and no iterative solving is required for these coupling variables. Therefore, the global optimization solving process is efficient whereas the solving accuracy is acceptable.

This paper is organized as follows. Section 2 describes the flowchart and architecture of the proposed SPD method. Section 3 presents the clustering process based on degree of coupling between different disciplines. In Sections 4 and 5, the decoupling process in a subsystem and the local optimization process are detailed, respectively. The whole approach is demonstrated and evaluated using three experiments introduced in Section 6. Finally, some conclusions and discussions are given in Section 7.

\section{Overview of the SPD method}

Compared to the existing architectures, the most obvious feature of the SPD method is that it contains three layers of components: the global optimizer on the top, the subsystem solver in the middle and the local optimizer on the bottom. Data are transferred among the three layers of components in a specific order. The main advantages of the proposed SPD method include: (1) the coupling variables that need to be decoupled have been decreased to a great extent by introducing the subsystem mechanism since the number of tasks to be processed is less than that of the traditional approaches; (2) a balance in the scales of different subsystems is attained by using the clustering analysis strategy for the coupling variables, which ensures that the time cost for solving each subsystem will not be much different; (3) only one local optimizer is constructed for each subsystem in order to control the complexity of local optimization problem, which can be constructed automatically and solved in an efficient way. Meanwhile, no additional dependent variables are required on the global optimizer, which ensures high solution efficiency.

\subsection{SPD flowchart}

As mentioned above, the core idea of the SPD method is to conduct data transfer and problem solving based on the three-layer architecture. In each time of iteration, the clustering analysis operation is executed on the whole coupling system and several subsystems are generated. Bidirectional interactions are conducted between the global optimizer and the subsystems while data are transferred in a unidirectional way within each subsystem. The SPD flowchart for solving MDO problems is given in Fig. 1, which starts with system initialization by setting the global 
design variables, the local design variables for each discipline and the interdisciplinary coupling variables. For the sake of clarity, a number of terms are used in this study: the coupling variable decoupled among the subsystems is called the global decoupling coupling variable (GDCV); the coupling variable decoupled in one subsystem is called the local decoupling coupling variable (LDCV); and the coupling variable without being decoupled at time of each iteration is called the reserved coupling variable (RCV).

After the system is initialized, four main steps need to be taken in the method:

a) Disciplinary analysis: conducting the disciplinary analysis for each discipline and updating the output variables;

b) Clustering analysis: executing the multidisciplinary clustering analysis as follows:

b.1) Calculating the sensitivity values between each discipline and each global optimization objective;

b.2) Conducting the clustering analysis based on the sensitivity value and then some subsystems are obtained. At the same time, adding the corresponding constraints of GDCVs to the global optimizer to attain coupling consistency among subsystems.

c) Subsystem optimization: optimizing all the subsystems as follows.

c.1) executing the serialization operation in each subsystem;

c.2) analyzing the subsystem and solving each output variable in the subsystem in a sequence according to the directed graph structure;

c.3) conducting the local optimization operation for the whole LDCVs in each subsystem.

d) Global optimization: optimizing the whole system globally and generating the new design variable values;

Step $b$ is repeated until the optimization process converges or the largest number of iteration has been reached.

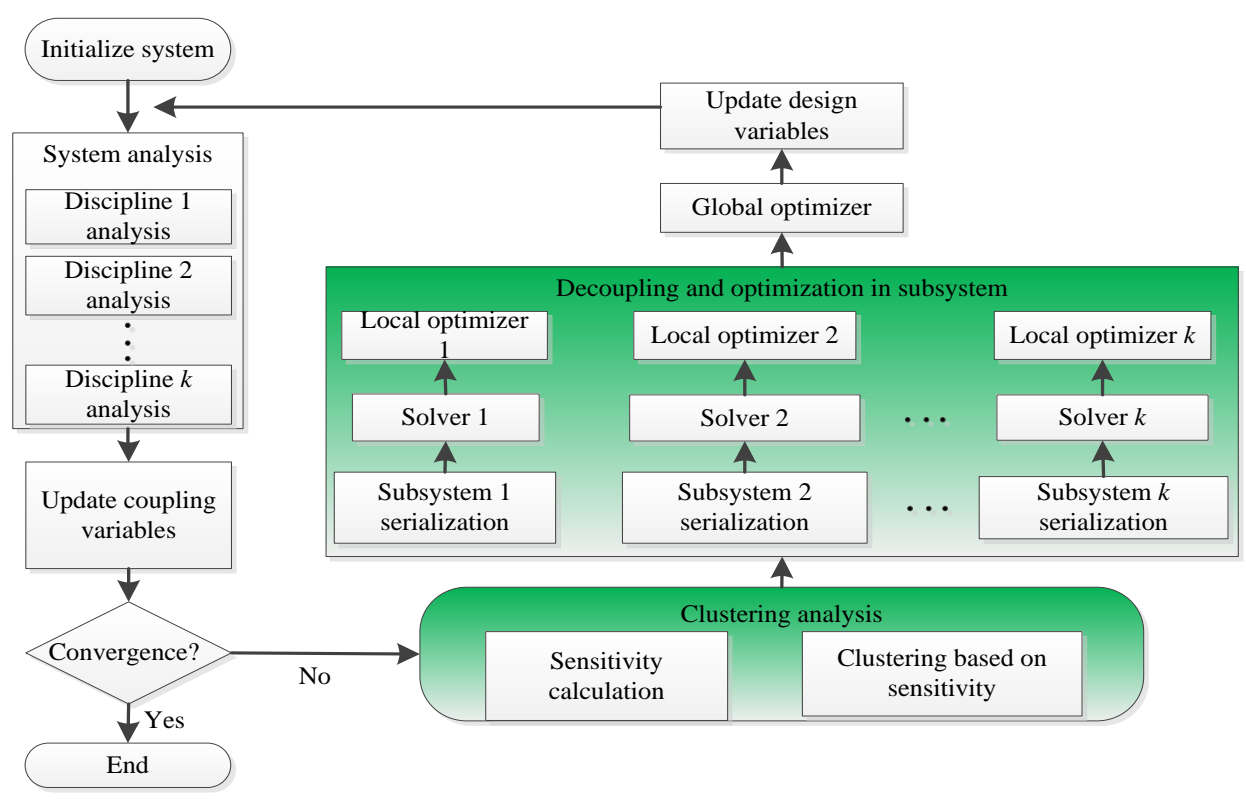

Fig. 1 Flowchart of SPD

The framework of SPD is shown in Fig. 2. 
the global optimizer managing the optimization of global objectives and the middle level is the subsystem solver that is responsible for the internal decoupling and discipline analysis. The bottom level is the local optimizer that is responsible for handling the LDCVs that need to be decoupled. This clear structure enables reasonable and effective processing on each variable.

Such a framework allows the SPD method to have the advantages of concurrent analysis and design while in the MDF architectures each subsystem only has one solver. Similar to those in IDF, the interactions between the global optimizer and each subsystem in SPD contain both design variables and the decoupling variables among subsystems. The global optimizer serves the purpose of coordinating the subsystems through these interactive coupling variables. Therefore, the subsystems are independent with each other and in this case parallel computing techniques can be applied.

The global optimizer selects the local design variables $\boldsymbol{x}_{k}$, the global design variables $\boldsymbol{x}_{s}$ and the coupling variables $\boldsymbol{y}_{\text {subsystem } \_k} i$ among the subsystems as the inputs for each subsystem. Each subsystem sends the objective values $\boldsymbol{f}_{k}$, the constraint values $\boldsymbol{g}_{k}, \boldsymbol{h}_{k}$ and coupling values $\boldsymbol{y}_{\text {subsystem_k_o }}$ back to the global optimizer as outputs. These variables are represented as black solid line between the global optimizer and the subsystem solvers. It should be noted that each subsystem remains a large number of coupling variables such as $\boldsymbol{y}_{31}$, $\boldsymbol{y}_{32}$ and $\boldsymbol{y}_{21}$ shown in black lines in subsystem 1 . To deal with the coupling variables in the subsystems, additional target variables are added into the global optimizer and the latter delivers the target variables to each local optimizer according to the dependency relationships. For instance, the target variable Subsystem_1_target is delivered to local optimizer $k$ in green dashed line. Meanwhile, the local optimizer delivers the dependent coupling variables to the subsystem such as Subsystem_k_coupling_var in black dashed line. Because of the unidirectional depending structure of the coupling variables in the subsystem, the disciplines in a subsystem can be solved according to its dependency relationships, which can ensure that the analysis process is executed only one time and can thus decrease the data transfer between the components to a great extent.

Based on the above analysis, the advantages of this framework can be summarized as follows. Firstly, the number of final subsystems is far less than the disciplines after executing the clustering analysis. Therefore, the number of local optimizers required is pretty small. Secondly, each subsystem can be analyzed rapidly through the serialization operation since only one time of analysis is required. Specifically, the differences between SPD and other traditional architectures are as follows:

(1) Compared to MDF, although some constraints are added for SPD in the search space, it is unnecessary to conduct iterative multidisciplinary analysis at the subsystem level. The execution efficiency can be improved, which is important for the coupling situations with more loops. 


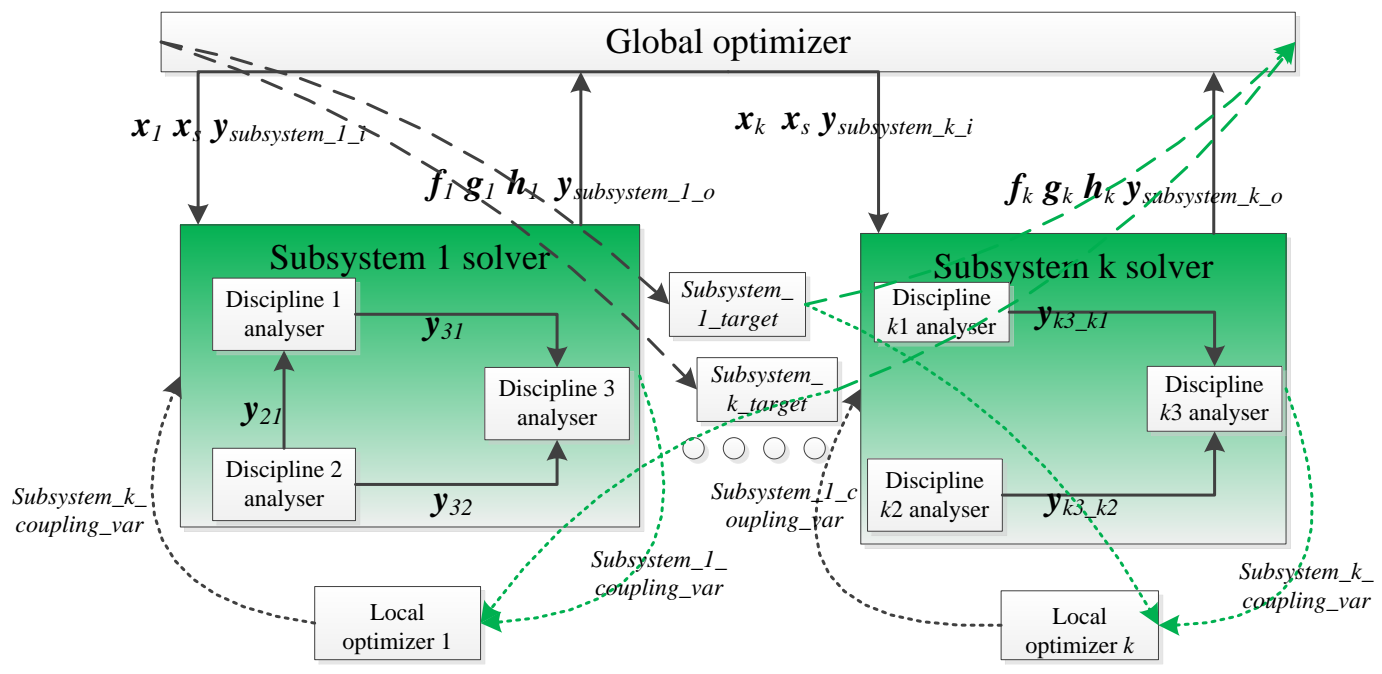

Fig. 2 The SPD framework

(2) Compared to IDF, the coupling variables that SPD needs to process is much less, most of which remain unchanged. It is a target decoupling strategy and thus the number of constraints added into the search space is far less than that of IDF. As a result, the scale of the global optimizer will not increase greatly. In this sense, it is more flexible in the search space and more satisfactory solutions can be acquired.

(3) Compared to the architectures with bi-level optimizers, the most outstanding advantage of SPD is that the number of local optimizers decreases a lot by constructing the subsystem components efficiently and reasonably. Meanwhile, the rapid subsystem analysis is executed and thus the efficiency of solving for all the subsystems is improved as a whole.

\section{Clustering Analysis Based on Sensitivity}

The degree of coupling among the disciplines involved and the global objective is calculated in this study, which is called coupling sensitivity. The existing approaches pay little attention to coupling sensitivity and often ignore some connotative information. With regard to SPD, a combination of the coupling sensitivity and a clustering strategy is adopted, that is, a multidisciplinary system is clustered into several subsystems according to the coupling sensitivity values obtained. Each subsystem may contain one or several disciplines and execute data transfer with the global optimizer. It is noteworthy that the subsystems are independent with each other and thus they can be executed simultaneously.

\subsection{Sensitivity analysis}

In MDO, sensitivity analysis refers to the analysis of system performance by evaluating its degree of being sensitive due to the changes in design variables or parameters. By performing in-depth analysis of the sensitivity information, the impacts of system design variables on the objectives or constraints can be identified. The coupling value between the subsystems can also be determined.

The sensitivity between a design function and a design variable represents the degree of changes in the design function caused by the changes in the design variable at a specific point, which can be described using the partial derivatives of the function. At a specific design point $X_{P}$, the sensitivity of the design function $\varphi_{i}(\mathrm{X})$ with respect to a specific design variable $x_{j}$ is thus defined as follows: 


$$
S_{i j}=\left.\frac{\partial \varphi_{i}(\mathrm{X})}{\partial x_{j}}\right|_{X=X_{P}}, i=1,2, \ldots, m, j=1,2, \ldots, n
$$

In this expression, $m$ and $n$ are the numbers of design functions and design variables, respectively. The larger the value of $\left|S_{i j}\right|$, the more sensitive the design function $\varphi_{i}(\mathrm{X})$ with respect to $x_{j}$, i.e., $x_{j}$ has a larger impact on $\varphi_{i}(\mathrm{X}) \cdot S_{i j}$ represents the monotonicity of function $\varphi_{i}(\mathrm{X})$ with respect to variable $x_{j}$. If $S_{i j}<0$, it means that $\varphi_{i}(\mathrm{X})$ is monotonically decreasing with respect to $x_{j}$. Otherwise, it is monotonically increasing. Therefore, the sensitivity value expresses two kinds of information: monotonicity and degree of being sensitive [24].

The sensitivity of design function $\left|S_{i j}\right|$ is employed to define the degree of coupling. The larger the value of $\left|S_{i j}\right|$ is, the stronger the coupling degree will be.

\subsection{Subsystem clustering based on sensitivity}

The idea that a complex engineering system is divided into several subsystems can be regarded as an abstract description of the problem using smaller and more manageable sub-problems. Each discipline contains local variables, global variables, output variables and its dependent input variables from other disciplines. The input variables from other disciplines are the interdisciplinary coupling variables. Two rules are mainly considered when the clustering analysis is conducted. Firstly, the subsystem coupling degree should be as low as possible, i.e. decreasing the dependencies between subsystems as much as possible. Also, there are no additional constraints for the coupling degree inside one subsystem. Secondly, the coupling degree between any subsystem and the global objective should be as low as possible. In this way, it can be ensured that the impacts of each subsystem with regard to the global objective are uniform and balanced. The traditional clustering problems usually involve several features, i.e. the dimension of each point and its values are represented on the absolute coordinate system. However, the distances between a point and the other points are known for the clustering problems based on sensitivities among the disciplines, which can be considered as a representation based on the relative coordinate system. Based on the above analysis, the initial centroid must belong to the current point set. In this study, an adapted $K$-means clustering algorithm is proposed to minimize the sum of the distances in each cluster. Obviously, different subsystem partitions lead to different decoupling variables, and thus have large impacts on the final subsystem sensitivities. To maximize the sensitivities among all the subsystems, it is represented as follows:

$$
E=\frac{\sum_{i=1}^{M} \sum_{j=1}^{N} \sum_{k=1}^{P} S_{j k}^{i}}{M}
$$

Specifically, $E$ represents the average subsystem sensitivities; $M$ and $N$ are the numbers of subsystems and disciplines respectively; $P$ is the number of disciplines in each subsystem. It can be seen that the representation is simple and can precisely reflect the distance between the subsystems compared to the standard distance measure used in the traditional $K$-means method. The adopted clustering strategy in this study is $K$-means algorithm combined with the global objective. Suppose that the allowed times of iteration is $T$, then the clustering process is as follows: 
a) Calculate the sum of global objective sensitivities with respect to all the coupling variables at the $k$-th time of iteration and mark it as $S$. The global objective sensitivity with respect to each subsystem is then $S / M$, represented as $A v g S$. Construct a container with $M$ elements, in which each element records the global objective sensitivity (with an initial value of 0 ) with respect to each subsystem. Select $M$ points whose sensitivities with respect to other points are not the largest as the initial centroids to ensure that the final result is not the worst;

b) For each left point $x_{i}$ in the point set, calculate its sensitivity with respect to all of the subsystems and select the subsystem with the biggest value. At the same time, calculate the corresponding global objective sensitivity value in the container. If it is bigger than $A v g S$, abandon the current selection and consider the subsystem with the second best sensitivity;

c) Calculate the current subsystem sensitivity value and re-allocate new centroids;

d) Repeat Steps $b$ and $c$ until $k$ reaches the maximal number of allowed iteration $T$, or the condition $\left\|E^{(\mathrm{k})}-E^{(\mathrm{k}-1)}\right\|<\Delta \quad$ is satisfied.

The selection of initial centroids has a large impact on the final clustering result in clustering algorithms. In this study, the selection strategy can to some extent ensure that good quality can be achieved for the final result. The reason for this is that for the problems in this study the number of disciplines is not too large and thus the number of clustering points is not large.

\section{Decoupling of a Subsystem}

After the clustering operation is conducted, each subsystem is highly correlated as several coupling loops exist. To improve the subsystem solving efficiency, SPD is executed to remove the internal coupling using the strategy of loop removal in a directed graph and serialize the disciplines in a subsystem. After that, the solver for the subsystem can execute the analysis process in an efficient way without the need of conducting iteration.

\subsection{Connection path between disciplines}

A subsystem is composed of one or more disciplines after the clustering analysis, in which the disciplines are highly correlated and dependent on each other. There may be several coupling variables with a large coupling degree in each subsystem. Moreover, it is also possible that there is a number of coupling loops in a subsystem. It is time-consuming to perform analysis for each subsystem during an optimization process. In this study, not all of the couplings between the disciplines are considered to be decoupled. Only a part of them with lower coupling values are considered to serialize all the disciplines in the same subsystem to solve the subsystem in a serializable way without the need of conducting iteration. Based on the above analysis, a loop removal method in directed graphs is adapted in this study with its details explained in the next paragraph.

Each discipline is regarded as a vertex in the directed graph based representation of a subsystem. The coupling variable between two disciplines means there exists one dependency and can be regarded as one directed edge. As shown in Fig. 3, two edges exist between discipline 1 and discipline 4, which means there are bidirectional couplings between these two disciplines. The output variables of discipline 4 are required when discipline 1 is analyzed and vice versa. If there is a directed path between $A$ and $B$ in a directed graph, there exists a connection path between $A$ and $B$. The number of this kind of directed paths is called the degree of a connection path. As shown in Fig. 3, no connection path exists from discipline 4 to discipline 3 whereas there 
is a connection path from discipline 3 to discipline 4 and the degree of connection path is 3. Specifically, three directed paths exist, namely 3->1->4, 3->2->4 and 3->1->2->4.

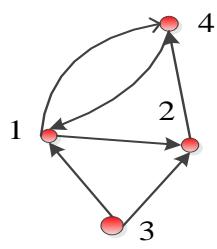

Fig. 3 An example of connection paths

\subsection{Serialization based partial decoupling process in a subsystem}

4.2.1 The general process of partial decoupling based on serialization

In this study, the breadth-first search method is used to conduct the serialization process. A list called vertex state list (VSL) is required to record the states of vertices in the directed graph. The sensitivity analysis information is taken into consideration when an edge is required to be removed in a loop, that is, the edge with the weakest sensitivity is removed so as to decrease the total influence to the largest extent. The input for this algorithm is a directed graph with loops while its output is a directed graph with no inner loops. The detailed process is as follows:

a) Select the vertex with the biggest in-degree as the initial vertex and insert it into the VSL;

b) Select the next untreated vertex as the current point in the VSL and find all of the connection paths from the current vertex to the initial vertex. In addition, the vertices dependent on the current vertex are also found, which are called dependent vertices;

c) For each dependent vertex, insert it into the list if it has not been inserted into the VSL; otherwise, check whether this dependent vertex lies on one or more connection paths. If only one connection path exists, compare the absolute values of the sensitivity of the directed edges and remove the directed edge with the weakest value in order to reserve the strong couplings in the subsystem. Another case is the one having more than one connection paths, i.e. the number of coupling loops is more than one. In this case, all the shared edges need to be found out and the one with weakest sensitivity absolute value should be removed. Only one edge is required to be removed through this strategy and all the coupling loops involved are broken;

d) Repeat steps $b$ and $c$ until all of the vertices in the VSL are processed.

\subsubsection{Formal demonstration of loop removal}

To provide a formal demonstration of the effectiveness of the SPD approach, the mathematical reasoning process is given as follows. Suppose that there is a directed graph $G$, marked as $G=(V, A)$, which is composed by a non-null limited vertex set $V$ and some ordered pair set $A$. Each element of $V=$ $\left\{v_{1}, v_{2}, \ldots, v_{n}\right\}$ is called a vertex of $G$. Another set $A=\left\{a_{1}, a_{2}, \ldots, a_{m}\right\}$ is called the edge set of $G$. Each element $a_{i j}=\left(v_{i}, v_{j}\right)$ is called the edge from vertex $v_{i}$ to vertex $v_{j}$, which corresponds to a coupling relationship in a real system. Additionally, $N_{i}$ represents the neighbor vertex set depending on $v_{i}$, from which there exists one edge pointing to $v_{i}$.

A directed loop is a loop whose edges have the same direction. This characteristic can ensure that it can be judged whether there is one directed loop correctly combining the proposed strategy between two vertexes. The mathematical reasoning process of loop removal between vertex $v_{i}$ and $v_{j}$ is as 
follows:

a) $\quad v_{i}$ has already been processed whereas $v_{j}$ has not, as shown in Fig. 4a. Moreover, there is not one edge from $v_{j}$ to $v_{i}$, which means $v_{j}$ does not depend on $v_{i}$. At this moment, it cannot be concluded that no directed loop exists containing vertexes $v_{i}$ and $v_{j}$.

b) When $v_{j}$ becomes the vertex currently processed as indicated with green color, its neighbor vertices can be obtained, i.e. $N_{j}$ that consists of vertices $v_{n}$ and $v_{i}$ both indicated with red color in Fig. 4a. This means that vertexes $v_{i}$ and $v_{n}$ alike are connected to $v_{j}$ with an edge element $a_{i j}$. Vertex $v_{i}$ will be processed as a dependency vertex of $v_{j}$.

c) Finally, one (the case in Fig. 4a) or more (the case in Fig. 4b) connection paths are found from $v_{j}$ to $v_{i}$. For the case in Fig. 4a, all the edges involved in the loop are indicated with green color and the edge from $v_{j}$ to $v_{l}$ is removed because of its weakest sensitivity absolute value. Two edges are considered for the case in Fig. $4 \mathrm{~b}$ and the shared edge from $v_{i}$ to $v_{j}$ is removed because of its weakest value. It can be seen that if this edge is broken, it can be assured that all the directed loops will be broken. This strategy can break up all the directed loops between the current vertex $v_{j}$ and the element of $N_{j}$. Furthermore, all of the directed loops can be broken up between any two vertices in $G$.

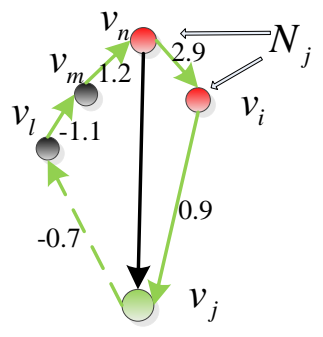

(a)

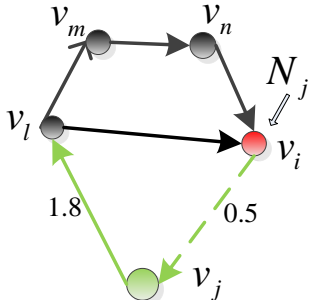

(b)
Fig. 4 A formal demonstration of loop removal

\subsubsection{Illustration of the whole SPD process}

Fig. 5 illustrates the algorithm in detail. Fig.5a shows the algorithm's inputs which include 7 vertices and 13 directed edges. In terms of the different colors used, red vertices are treated; green vertex is the current vertex; grey vertices are untreated; and purple edges are removed edges. The serialized process is detailed as follows.

a) Vertex 4 is selected as the initial vertex since it has the biggest in-degree and insert it into the VSL, as shown in Fig. 5b;

b) Select vertex 4 as the current vertex, find all its dependent vertices, which are vertices 1, 2 and 3 in this case. Insert them into the VSL and mark vertex 4 as treated, as shown in Fig. 5c;

c) Select vertex 1 as the current vertex, insert its dependent vertices 5 and 6 into the VSL and mark it as treated, as shown in Fig. 5d;

d) Select vertex 2 as the current vertex which has two dependent vertices, namely vertices 1 and 6 . However, they are already in the VSL, it is unnecessary to do the inserting operation again. There is only one 
connection path from vertex 2 to vertex 4 , namely 2->4. Moreover, both of vertex 1 and vertex 6 are not in this connection path. The directed edges from these two vertices to vertex 2 can be added into the directed graph. Mark vertex 2 as treated, as shown in Fig. 5e;

e) Select vertex 3 as the current vertex, insert vertex 7 into the VSL. Obviously, there is only one connection path from vertex 3 to 4 and therefore the directed edge from vertex 2 to 3 can be reserved. In addition, vertex 1 depends on vertex 3 and it has already been marked as treated. However, there is no connection path from vertex 3 to 1 , which means no directed loop containing vertexes 3 and 1 exist. Therefore, no edge is required to be removed. Marked vertex 3 as treated, as shown in Fig. 5f.

f) Select vertex 5 as the current vertex. According to their sensitivity values, the directed edge from vertex 1 to 5 is removed because of smaller sensitivity values. Marked vertex 5 as treated, as shown in Fig. 5g;

g) Select vertex 6 as the current vertex. It can be seen that vertex 3 depends on it and there are two connection paths from vertex 6 to the vertex 3 (6->1->2->3, 6->2->3).In this case, the directed edge from vertex 3 to 6 is removed, resulting in the breaking up of two coupling loops. Mark vertex 6 as treated, as shown in Fig. 5h;

h) Select vertex 7 as the current vertex. It can be seen that vertex 4 depends on it. However, there is a connection path from vertex 7 to 4 , namely 7->3->4. Thus, the directed edge from vertex 7 to 3 is removed because of its smallest absolute sensitivity value. Mark vertex 7 as treated, as shown in Fig. 5j.

Finally all the vertices are treated. Fig. $5 \mathrm{j}$ is the final directed graph obtained as the output of the algorithm.

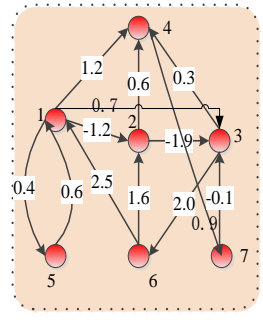

(a)

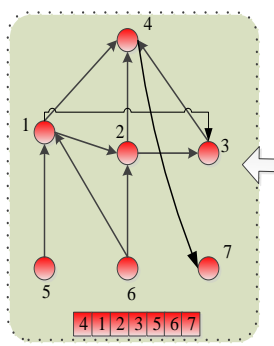

(j)

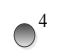

4
(b)

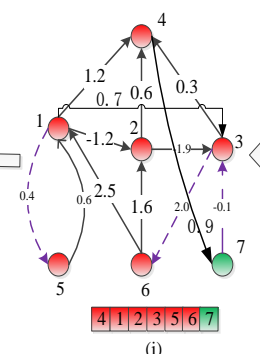

(i)
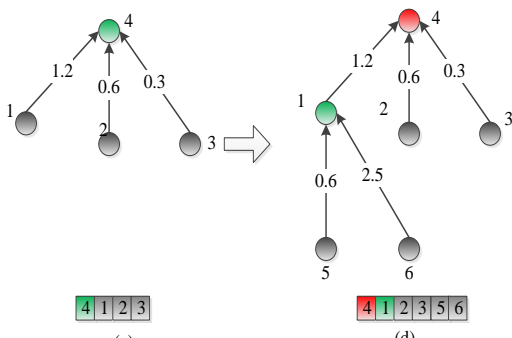

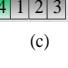
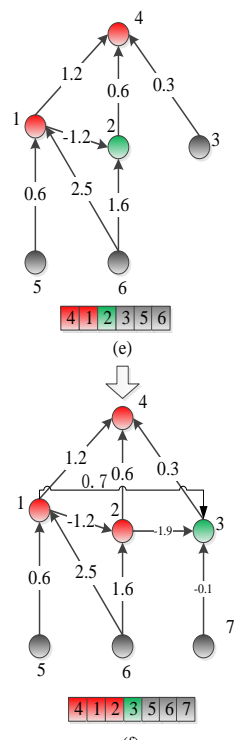

Fig.5 The serialization process in a subsystem

algorithm of the serialized directed graph is as follows:

\subsection{Serializable solving in a subsystem}

After the serialization operation is completed, the solution sequence is also determined for each subsystem. The solving a) Calculate the out-degree for each vertex in the serialized directed graph;

b) Find the vertex whose out-degree is 0 , put 
it into the sequence solving list and decrease all of the out-degree of its dependent vertices by one;

c) Repeat Step (b) until all the vertices have been placed in the solving sequence list.

Take Fig. $4 \mathrm{j}$ as an example, the calculation process is detailed as follows:

a) The out-degree of vertex 7 is 0 , put it in the solving sequence list and decrease the out-degree of vertex 4 by one.

b) The out-degree of the initial vertex 4 is 0 , put it in the solving sequence list and decrease the out-degree of vertices 1,2 and 3 by one;

c) The out-degree of vertex 3 is 0 and put it in the solving sequence list. Decrease the out-degree of vertices 1 and 2 by one;

d) The out-degrees of vertex 2 is 0 , thus it is put in the solving sequence list. Decrease the out-degree of vertices 1 and 6 by one;

e) The out-degrees of vertex 1 is 0 and thus they are put in the solving sequence list. Decrease the out-degree of vertices 5 and 6 by one;

Put vertices 5 and 6 in the solving sequence list. The final solving sequence list is then obtained as (7-4-3-2-1-6-5). The subsystem solver is executed according to this sequence without the need to perform iteration

Note that many coupling variables among different disciplines can be reserved by using the serialized decoupling strategy. As shown in this example with initial 13 coupling variables, 10 coupling variables are reserved after the subsystem decoupling operation has been executed. The subsequent local optimizer only needs to deal with three decoupled variables. Compared to IDF's operation of adding 13 constraints to be considered by the global optimizer, the proposed SPD is much more efficient in terms of search space exploration. Moreover, each subsystem solver only run one time whereas in MDF three iteration-based analysis will be required. This shows that solving efficiency is significantly improved. In particular, for the system with more complicated coupled relationships, the performance improvement achieved by SPD is much more prominent.

\section{Local optimization in a subsystem}

As mentioned above, most of the coupling variables can be analyzed serially after the previous step is executed. A small part of variables need to be decoupled. These variables can be regarded as design variables and some corresponding constraints are appended in the global optimization model. However, it will possibly make the global optimization model to be excessively constrained and as a result the search space is greatly narrowed down.

In this study, a local optimization method is proposed to handle the coupling variables within a subsystem and keep the whole system consistent. In this case, the key issue is how to ensure the efficiency of solving the global optimization model and the acceptance of local dynamical optimization models. Meanwhile, it is also important to deal with the related decoupled variables in a reasonable way. Based on the above analysis, the construction of a proper local optimization model is necessitated. As shown in Fig. 6, for each time of iteration, the bottom local optimizer accepts the target variables from the global optimizer and the output variables of its corresponding subsystem. The decoupled variables in each subsystem are regarded as the optimization variables of a local optimization model. Each local optimizer is only conducted for its corresponding subsystem to solve the decoupled variables. Moreover, the coupling sensitivities of the decoupled variables for its corresponding subsystem are also taken into account since different decoupled variables make distinct impacts on the same subsystem. To precisely reflect the impacts on a local optimization 
model, the coupling sensitivities are calculated and appended to local optimization model. For the local optimization objective, it is the combination of the coupling sensitivities and the square sum of the difference between the decoupled variables and the target variables. The detailed process of constructing the local optimization process for the $i$ th subsystem is as follows:

a) Calculate the sensitivity $S_{i j}$ for the $j$ th decoupled variable. In Equation (3), $P_{i}$ is the number of coupling variables in the $i$ th subsystem and $S_{k}$ is the sensitivity between the $j$ th and $k$ th decoupled variable.

$$
S_{i j}=\sum_{k=1}^{P_{i}}\left\|S_{k}\right\|
$$

b) Normalize the values in the vector $S_{i}$ and obtain the vector $W_{i}$.

c) Construct the local optimization model using Equation (4).

$$
f_{i}=\sum_{j=1}^{Q_{i}} W_{i j}\left(\hat{y}_{j_{-} t}-y_{j_{-} t}\right)^{2}
$$

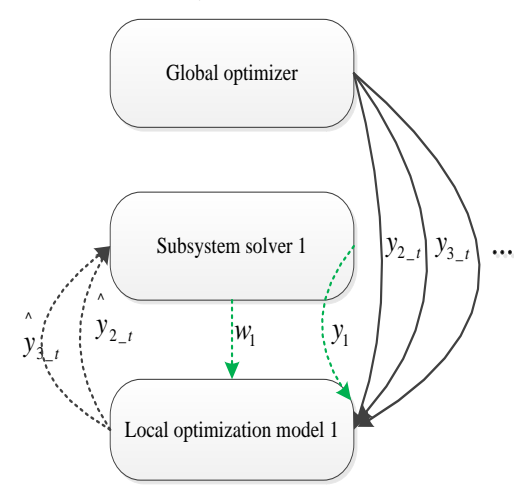

Fig.6 Construction of a local optimization model

In Equation (4), $Q_{i}$ is the number of decoupled variables in the $i$ th subsystem; $y_{j_{-} t}$ is the target variable delivered from the global optimizer, which is a constant in the local optimization model; and $y_{j_{-} t}$ is the local optimization variables. Some boundaries and local constraints may also be given. According to the characteristics of the local optimization model, the optimization method named square quadratic programming is used [25].

It should be noted that the global optimizer does not operate on the coupling variables in the internal subsystem directly whereas a corresponding target variable is appended. This strategy can ensure the components normally communicate without affecting the flexibility of the global optimizer and in this way search efficiency can be ensured. To avoid long running time caused by the inner loop during the local optimization operation, a maximum number of iteration value can be set.

The advantages of this proposed method include: (1) the subsystems can be solved independently and simultaneously since the local optimizer only communicates with its corresponding subsystem; (2) for a decoupled variable in a subsystem, a corresponding target variable is added into the global optimizer, which drives the coupling variables towards the optimal solution. Compared to the handling of inter-subsystem variables, the global optimizer is more flexible and efficient in terms of search space exploration; (3) each subsystem only contains one local optimization model and is dynamically constructed with a fast solving speed. These characteristics can help improve the overall performance.

\section{Computational experiments}

To demonstrate the efficacy and efficiency of the proposed SPD approach, three experiments of multidisciplinary optimization are constructed by using different mathematical models. These models have some representative features: (1) involving multiple variables; (2) involving multiple disciplines; and (3) being highly coupled. Moreover, the optimization results of SPD are compared to MDF and IDF since bi-level structural strategy is not considered in the proposed SPD in the current work. The 
experiments are conducted on the OpenMDAO [26] platform developed by NASA.

\subsection{Partial decoupling of a single subsystem}

The first typical test application is called the Sellar [27] problem which has a mathematical model as follows:

Minimize:

$$
f=x_{1}^{2}+z_{2}+y_{1}+e^{-y_{2}}
$$

Design variables: $x_{1}, z_{1}, z_{2}$

Subject to:

$$
\begin{array}{r}
y_{1}=z_{1}^{2}+x_{1}+z_{2}-0.2 y_{2} \\
y_{2}=\sqrt{y_{1}}+z_{1}+z_{2}
\end{array}
$$

In this example, two disciplines are entirely coupled through $y_{1}$ and $y_{2}$ even it looks simple. It is solved through three architectures, namely SPD, MDF and IDF. This problem is directly described as one subsystem, and thus clustering analysis is not required. In the SPD method, $y_{1}$ is decoupled and a local optimizer is constructed to deal with it. This experiment is used to demonstrate the effectiveness of partial decoupling and local optimization.

Table 1 shows the result of Experiment 1. It can be seen that IDF has the optimal result but requires the longest time. On the contrary, MDF's execution efficiency is better than the others but it has the worst results. The proposed SPD method lies between the two in terms of both efficiency and performance. Moreover, the final two coupling variables converge to the same position. The experimental results indicate that the proposed SPD method has good effectiveness in terms

\begin{tabular}{|c|c|c|c|}
\hline & SPD & IDF & MDF \\
\hline \multicolumn{4}{|c|}{ Optimal positions: } \\
\hline$z_{1}$ & 1.977670 & 1.977658 & 1.977639 \\
\hline$z_{2}$ & 0.0 & 0.0 & 0.0 \\
\hline$x_{1}$ & 0.0 & 0.0 & 0.0 \\
\hline
\end{tabular}
of the partial decoupling strategy.

Table 1. Results of Experiment 1

\begin{tabular}{rcccc}
$y_{1}$ & & 3.16 & 3.16 & 3.16 \\
$y_{2}$ & & 3.755309 & 3.755383 & 3.755278 \\
Optimal & $f$ & $\mathbf{3 . 1 8 3 3 9 3 2}$ & $\mathbf{3 . 1 8 3 3 9 1 4}$ & $\mathbf{3 . 1 8 3 3 9 3 7}$ \\
Runtime & $(\mathrm{s})$ & $\mathbf{0 . 5 5 5}$ & $\mathbf{0 . 6 4 1}$ & $\mathbf{0 . 2 5 1}$ \\
\hline
\end{tabular}

\subsection{Serializable decoupling of multidisciplinary problems}

To demonstrate the clustering analysis and serializable decoupling strategy, a large-scale complicated system is constructed with the help of CASCADE [28] and is shown below:

Minimize:

$$
f=x_{1}^{2}+z_{2}+y_{1}+e^{-y_{2}}+x_{41}-0.5 x_{8}+0.8 y_{3}-2.5 y_{4}+y_{6}^{2}
$$

Design variables: $x_{1}, x_{3}, x_{41}, x_{42}, x_{5}$,

$$
x_{8}, \quad z_{1}, z_{2}
$$

Subject to:

$$
\begin{aligned}
& y_{1}=z_{1}^{2}+x_{1}+z_{2}-0.2 y_{2} \\
& y_{2}=\sqrt{y_{1}}+z_{1}+z_{2} \\
& y_{3}=z_{1}+z_{2}^{3}+x_{3}-0.5 y_{1}+2 y_{5} \\
& y_{4}=z_{2}-x_{41}+0.5 x_{42}+y_{1}^{2}-y_{3} \\
& y_{5}=z_{1}+z_{2}-x_{5}+\sqrt{y_{2}}+2.5 y_{3}+y_{7} \\
& y_{6}=z_{1}-y_{4}^{2}+y_{8} \\
& y_{7}=z_{1}-z_{2}+y_{1}+0.2 y_{6}-3 y_{9} \\
& y_{8}=z_{1}+z_{2}-x_{8}+1.5 y_{2}+\sqrt{y_{5}} \\
& y_{9}=z_{1}+y_{4} \\
& \hline 1+4 \\
& \hline 4
\end{aligned}
$$

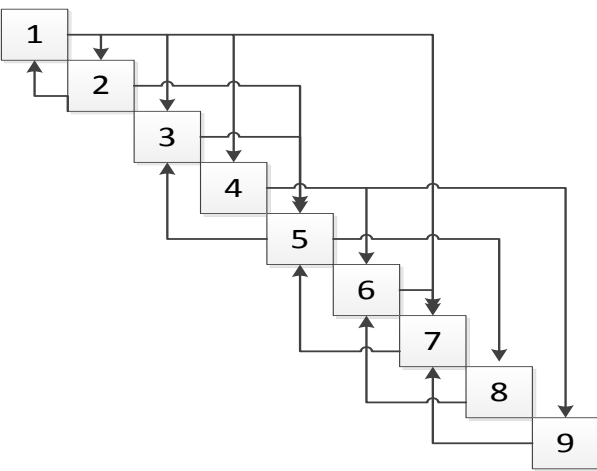

Fig. 7 The design structure matrix of the case in Experiment 2

This optimization problem is composed of 2 global design variables, 8 local design variables, 2 global constraints, 9 logical disciplines and 17 coupling variables. Fig. 7 gives the design structure matrix of this problem to help more clearly understand the coupling relationships and sequences. It can be seen that each discipline has one output 
variable in this case.

The numerical calculation process in this experiment converged within 9 times of iteration, with the numbers of GDCVs, LDCVs and RSVs changing dynamically for each time of iteration. Fig. 8 shows the changes of the quantities of these variables. It can be seen that the values of the quantities keep changing and achieve convergence gradually. Moreover, the quantity of RCVs is obviously more than that of the others, approximating to 10 on average. This indicates that the SPD method can ensure a majority of coupling variables is reserved during the execution process for this complex mathematical optimization model. Only a small percentage of coupling variables are required to be decoupled and processed, and the solving efficiency is satisfactory compared to other solving architectures ascribed to this characteristic.

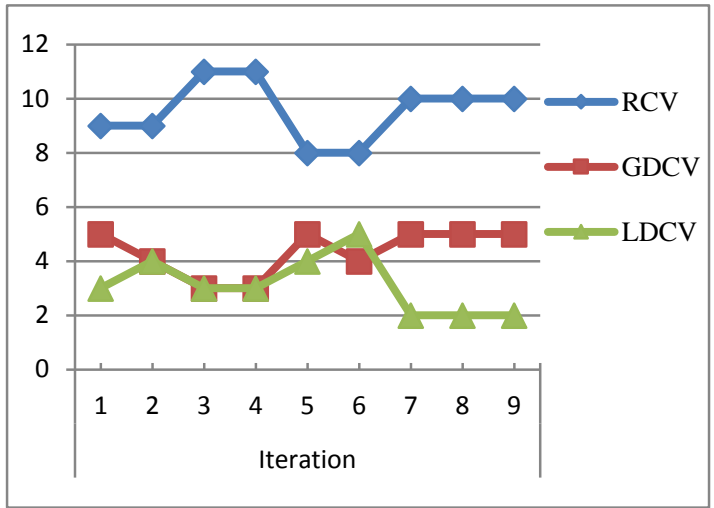

Fig. 8 Changes of the quantities of coupling variables in Experiment 2

Table 2 lists the results using SPD, IDF and MDF. The optimal of the SPD method is -17.295641 whereas it is -13.925012 for IDF. Thus the performance of the proposed SPD method is better. The time cost of SPD is 13.112 seconds whereas it is 22.654 seconds for IDF. When the SPD arrives at the optimal position, its design variables $x_{3}$ and $x_{5}$ have large differences from those in IDF and are much further away from the initial positions, which indicates that exploration of the search place in the SPD method is better and the objective is not restricted in the local optimal.

This indicates that IDF is not quite suitable for highly coupled systems since a large number of constraints are added for it. MDF get some similar results. However, when this problem is solved by using SPD, the system is clustered into some subsystems and thus the constraints added to the global optimizer are decreased to a great extent.

Table 2. Results of Experiment 2

\begin{tabular}{|c|c|c|c|}
\hline & SPD & IDF & MDF \\
\hline \multicolumn{4}{|c|}{ Optimal positions: } \\
\hline$z_{1}$ & 2.522890 & 2.601791 & 2.714256 \\
\hline$z_{2}$ & -2.716901 & -3.336445 & -2.537815 \\
\hline$x_{1}$ & -0.182680 & -0.061898 & -0.102563 \\
\hline$x_{3}$ & 63.818679 & 16.981197 & 17.368944 \\
\hline$x_{41}$ & -10 & -10 & -10 \\
\hline$x_{42}$ & -10 & -10 & -10 \\
\hline$x_{5}$ & 27.980476 & 18.772161 & 17.256341 \\
\hline$x_{8}$ & -8.797885 & -8.785601 & -8.745231 \\
\hline \multicolumn{4}{|c|}{ Coupling variables: } \\
\hline$y_{1}$ & 3.160445 & 3.160084 & 3.160245 \\
\hline$y_{2}$ & 1.586152 & 1.0431 & 1.23564 \\
\hline$y_{3}$ & -2.046238 & 1.954615 & 1.123701 \\
\hline$y_{4}$ & 4.305998 & 4.044348 & 4.120124 \\
\hline$y_{5}$ & -23.649674 & -7.73309 & -6.124501 \\
\hline$y_{6}$ & -0.160549 & -0.222563 & 0.154714 \\
\hline$y_{7}$ & 8.370595 & 3.114453 & 2.478012 \\
\hline$y_{8}$ & 15.848595 & 15.133312 & 15.452412 \\
\hline$y_{9}$ & 6.831288 & 5.039413 & 5.1234514 \\
\hline Optimal $f$ & -17.295641 & -13.925012 & -14.015410 \\
\hline Runtime（s） & 13.112 & 22.654 & 24.531 \\
\hline
\end{tabular}

\subsection{Problem with multiple couplings in an} engineering case

A real engineering example [29] involving complex coupling relationships is studied in Experiment 3. In particular, multiple couplings exist between two of the disciplines involved in this example. In this case, a combination of two simple multidisciplinary problems in MODEL of Bullalio University is adopted 
[30]. As shown in Fig. 9, it is composed of the combustion of propane problem and the Golinski's speed reducer problem. The combustion of propane problem contains 3 disciplines, 11 design variables, 11 coupling variables and 2 fixed parameters whereas another problem contains 2 disciplines, 7 design variables, 4 coupling variables and 7 fixed parameters. A coupling variable $y_{12}$ is constructed to combine the two problems.

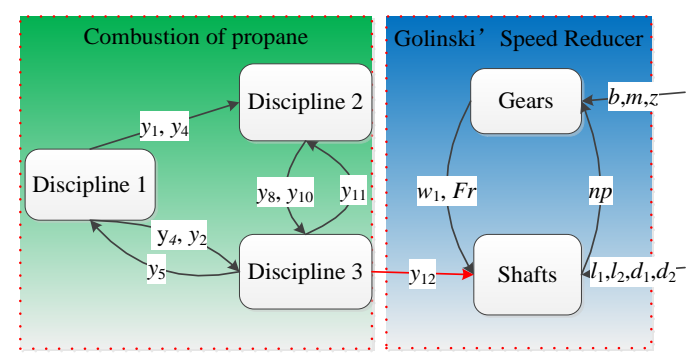

Fig. 9 Coupling relationships of the problem solved in Experiment 3

The detailed optimization model is given as follows:

Minimize: $f=y_{2}+y_{6}+y_{7}+y_{9}+W$

Design variables: from $x_{1}$ to $x_{11}, b, m, z, l_{1}, l_{2}$, $d_{1}, d_{2}$

Parameters: $p, R, P, r, K g, P d, q, K g_{1}, K g_{2}$ Coupling relationships:

$$
\begin{aligned}
& y_{1}=x_{1}+x_{4}-3 \\
& y_{2}=2 x_{1}+x_{2}+x_{4}+x_{7}+x_{8}+x_{9}+2 x_{10}-R \\
& y_{3}=2 x_{2}+2 x_{5}+x_{6}+x_{7}-8 \\
& y_{4}=2 x_{3}+x_{9}-4 R \\
& y_{5}=K_{5} x_{2} x_{5} x_{4}-x_{1} x_{5} \\
& y_{6}=K_{6} \sqrt{x_{2} x_{4}}-\sqrt{x_{1}} x_{6} \sqrt{\frac{P}{x_{11}}} \\
& y_{7}=K_{7} \sqrt{x_{1} x_{2}}-\sqrt{x_{4}} x_{7} \sqrt{\frac{P}{x_{11}}} \\
& y_{8}=K_{8} x_{1}-x_{4} x_{8} \frac{P}{x_{11}}
\end{aligned}
$$

$$
\begin{aligned}
& y_{9}=K_{9} x_{1} \sqrt{x_{3}}-x_{4} x_{8} \sqrt{\frac{P}{x_{11}}} \\
& y_{10}=K_{10} x_{10}-x_{4} x_{10} \frac{P}{x_{11}} \\
& y_{11}=x_{11}-\sum_{i=1}^{10} x_{i} \\
& w_{1}=\left[\left(d_{s t}^{2}-d_{w}^{2}\right) \frac{\pi b}{4}+\left(d_{w}^{2}-d_{p}^{2}\right) \frac{\pi b}{12}+\left(d_{p}^{2}-d^{2}\right) \frac{\pi(2 \mathrm{~d})}{4}\right] * \text { density } \\
& F r=\frac{2 M}{m z} \tan (\alpha) \\
& n_{p}=\frac{60 w_{1}}{2 \pi} r m y_{12}
\end{aligned}
$$

In this engineering case, the quantities of RCV, GDCV and LDCV are shown in Fig. 10. It can be seen from the figure that the quantity of RCV takes the majority of the total quantity. This indicates that most coupling variables are reserved. The quantities of GDCV and LDCV are 1 or 2 , respectively, meaning that the global optimizer is not required to take much extra effort to explore the search space. Meanwhile, the scale of the local optimizer is not large and can be solved with a high efficiency. This experiment of the engineering case demonstrates that the coupling variables can be decoupled in a satisfactory way by using the proposed architecture.

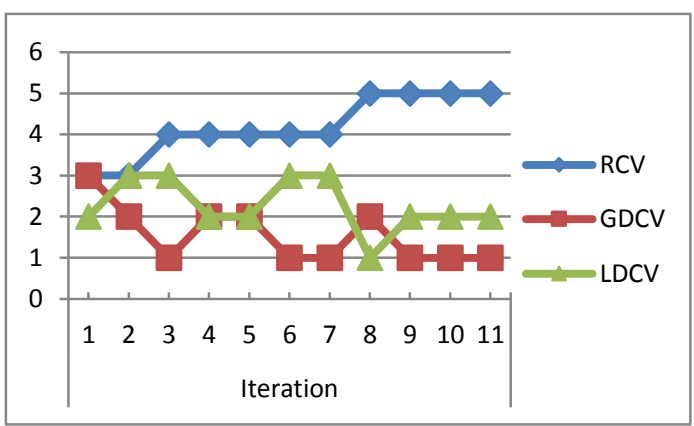

Fig. 10 Changes of quantities of coupling variables in Experiment 3

Table 3 lists the results obtained by solving the engineering problem in Experiment 3 using the SPD, IDF and MDF methods. The performances comparison helps draw a very similar conclusion to the one got in the previous experiment. Specifically, the 
MDF architecture is suitable for large-scale optimization model without complicated coupling relationships since the complete interdisciplinary analysis needs to be performed for each time of iteration. It can be seen through these two cases that the proposed SPD method is both feasible and practical for the problems with multiple complex couplings between any two disciplines.

Table 3. Results of Experiment 3

\begin{tabular}{cccc}
\hline \multicolumn{5}{c}{ SPD } & IDF & MDF \\
\hline \multicolumn{4}{c}{ Coupling variables: } \\
$y_{1}$ & 1.0241423 & 2.145101 & 1.891420 \\
$y_{2}$ & 2.142575 & 3.012784 & 2.014752 \\
$y_{3}$ & 1.454147 & 3.147852 & 3.2141025 \\
$y_{4}$ & 2.478235 & 4.147520 & 9.124014 \\
$y_{5}$ & -1.145741 & -0.145368 & -0.258142 \\
$y_{6}$ & -10.25413 & -8.528412 & -9.857142 \\
$y_{7}$ & 2.451368 & 4.254145 & 5.256985 \\
$y_{8}$ & 6.145865 & 5.254369 & 8.369145 \\
$y_{9}$ & 9.145268 & 12.235456 & 11.256354 \\
$y_{10}$ & 30.254124 & 26.142125 & 36.542114 \\
$y_{11}$ & -1.254147 & -1.89745 & -1.896521 \\
$y_{12}$ & 6.354896 & 8.567841 & 9.256341 \\
$F r$ & 2.014123 & 1.254156 & 1.568474 \\
Runtime $(\mathbf{s})$ & $\mathbf{3 5 . 1 4 5}$ & $\mathbf{5 4 . 2 1 4}$ & $\mathbf{5 7 . 2 3 5}$ \\
\hline$N p$ & 5.214145 & 6.154251 & 7.365840 \\
$W_{1}$ & 10.254786 & 15.234561 & 16.547369 \\
\hline Optimal $\boldsymbol{1 3}$ & $\mathbf{1 3 . 1 4 5 7 8 9}$ & $\mathbf{1 8 . 6 5 7 4 5 9}$ & $\mathbf{2 0 . 1 4 5 7 8 6}$ \\
\hline
\end{tabular}

\section{Conclusion and Future Work}

In this search, a SPD approach to solving MDO problems is proposed to achieve better performance in terms of complexity and efficiency. Different from existing approaches, the SPD method has a number of features:

(1) A three-layer framework is proposed, including the global optimizer, the subsystem solver and the local optimizer;

(2) A clustering strategy is proposed based on sensitivity analysis, through which the multidisciplinary system is decomposed into several subsystems with reasonable and balanced scales for the subsequent analysis;

(3) A serialization strategy is proposed for tacking the coupling relationships in a subsystem based on the directed graph theory. The main advantage of this strategy is that the solver for a subsystem can execute numerical analysis for a specific discipline with high efficiency while many coupling variables are reserved;

(4) A local optimization process is proposed for the internal decoupled issues within a subsystem. The internal decoupled issues can be properly solved by dynamically constructing a local optimization model with a very high running speed.

In this current work, some unsatisfactory issues have also been found. Firstly, it needs the derivative information, which inevitably affects the overall performance. Additionally, the clustering operation and the serialization process should be further improved for the MDO systems involving many disciplines. Future work will be focused on exploring the combination of serialization and suspension to attain better solutions.

\section{Acknowledgement}

The authors appreciate the financial supports from the NSF of China (61173126 and 61370182), the 863 High-Technology Project of China (2011AA100804) and Key Project of Science and Technology of Zhejiang Province (2014C01052).

\section{References}

[1] Joaquim R. R. A., Andrew B. L. Multidisciplinary Design Optimization: A Survery of Edgehitectures. AIAA Journal, 2013, 51(9):2049-2075.

[2] Huang C.H. Development of Multi-objective Concurrent Subspace Optimization and 
Visualization Methods for Multidisciplinary Design (Ph.D thesis). Department of Mechanical and Aerospace Engineering, State University of New York at Buffalio, April 2003.

[3] Mastroddi F., Gemma S. Analysis of Pareto Frontiers for Multidisciplinary Design Optimization of Aircraft. Aerospace Science and Technology, 2013, 28(1):40-55.

[4] Chebbah M. S., Naceur H., Hecini M. Rapid Coupling Optimization Method for a Tube Hydroforming Process. Proceedings of the Institution of Mechanical Engineers, Part B: Journal of Engineering Manufacture, 2010,224(2):245-256.

[5] Lin L., Liu M. Z., Tang J., Zhao Z. B., Ge M. G. The Coupling Relationship among Bottleneck Shifting Factors in Job Shop. Proceedings of the Institution of Mechanical Engineers, Part B: Journal of Engineering Manufacture, 2013, 227(9): 1373-1381.

[6] Zhang P., Chen X. A. Thermal-mechanical Coupling Model-based Dynamical Properties Analysis of a Motorized Spindle System. Proceedings of the Institution of Mechanical Engineers, Part B: Journal of Engineering Manufacture, 2014, 1-12.

[7] Sobieszezanski-Sobieski J.Leonard L., Sirkett D. M. Engineering a New Wrist Joint Replacement Prosthesis-a Multidisciplinary Approach. Journal of Engineering Manufacture, 2002, 216:1297-1302.

[8] Weck O. D., Agte J., Sobieszezanski-Sobieski J. State-of- the-art and Future trends in Multidisciplinary Design Optimization. In: Proceedings of the $48^{\text {th }}$ AIAA/ASME/ASCE/AHS/ACS Structures, Structural Dynamics, and Materials Conference, AIAA-2007-1905, 2007, April 23-26.

[9] Myers R. H., Montogomery D. C. Response Surface Methodology: Process and Product Optimization using Designed Experiments. New York: Wiley, 1995.

[10] Braun R. D., Kroo I. M. Development and Application of the Collaborative Optimization
Architecture in a Multidisciplinary Design

Environment. In Proceedings of the ICASE/NASA Langey Workshop on Multidisciplinary Design Optimization, Hampton, Virginia, 1995, 98-116.

[11] Zadeh P. M., Toropov V. V., Wood A. S. Metamodel-based Collaborative Optimization Framework. Structural and Multidisciplinary Optimization, 2009, 38(2):103-115.

[12] Sobieszezanski-Sobieski J, Chopra I. Multidisciplinary Optimization of Aeronautical System. Journal of Aircraft, 1990, 12: 977-978.

[13] Li Y., Lu Z., Michalek J. J. Diagonal Quadratic Approximation for Parallelization of Analytical Target Cascading. Journal of Mechanical Design, 2008, 130(5):051402-1-051402-11.

[14] Tappeta R. V., Renaud J. E. Multi-objective Collaborative Optimization. Journal of Mechanical Design, 1997, 119(3):403-411.

[15] Simpson T. W., Mistree F., Korte J. J. Kriging Models for Global Approximation in Simulation-based Multidisciplinary Design Optimization. AIAA Journal, 2001, 39(12):2233-2241.

[16] Han M. H.. Multidisciplinary Design Optimization Approaches and Technique Research for Complex Engineering Systems. Beijing University of Aeronautics and Astronautics.

[17] Yao W., Chen X. Q., Luo W. C. Review of Uncertainty-based Multidisciplinary Design Optimization Methods for Aerospace Vehicles. Progress In Aerospace Sciences, 2011, 47(6):450-479

[18] Wang D. H., Hu F., Ma Z. Y. A CAD/CAE Integrated Framework for Structural Design Optimization Using Sequential Approximation Optimization. Advances in Engineering Software, 2014,76:56-68.

[19] Patnaik S.N., Hopkins D.A. General Purpose Optimization Method for Multidisciplinary Design Applications. Advances in Engineering Software, 2000, 31:57-63.

[20] Sobieszezanski-Sobieski J. Sensitivity of 
Complex Internally Coupled Systems. AIAA Journal, 1990,28(1):153-160.

[21] Braun R. D., Kroo I. M. Development and Application of the Collaborative Optimization Architecture in a Multidisciplinary Design Environment. SIAM Journal, 1995, 98-116.

[22] Sell R. S., Batill S. M., Renaud J. E. Response Surface Based Concurrent Subspace Optimization for Multidisciplinary System Design. AIAA, 1996, 96-0714.

[23] Sobieszczanski-Sobieski J., Agte J. S. and Sandusky R. R. Bi-Level Integrated System Synthesis (BLISS). NASA/TM-1998-208715.

[24] Qiu Q. Y., Feng P. E., Dong L. G. Approaches for Analyzing the Optimization Results Based on the Sensitivity Information. Journal of Zhejiang university, 2000, 34(6):603-607.

[25] Irene R. L., Ramon H., Charles E. Quadratic Programming Feature Selection. Journal of Machine Learning Research, 2010,
11:1491-1516.

[26] Justin G., Kenneth T. M. Standard Platform for Benchmarking Multidisciplinary Design Analysis and Optimization Architectures. AIAA Journal, 2013, 51(10).

[27] Chan L. Evaluation of Two Concurrent Design Approaches in Multidisciplinary Design Optimization . NRC report LM-A-077, 2001.

[28] Hulme K. F., Bloebaum C L. Development of CASCADE- A Multidisciplinary Design Test Simulator. AIAA, 1996.

[29] Roy R., Hinduja S., Teti R. Recent Advances in Engineering Design Optimization: Challenges and Future Trends. CIRP Annals Manufacturing Technology, 2008, 57(2):697-715

[30] Parashar. S. Decision Support Tool for Multidisciplinary Design Optimization Using Multi-domain Decomposition. Master of Science Thesis, 2004,29-34. 